\title{
Achilles tendon rupture using FHL tendon transfer endoscopically
}

\begin{abstract}
Objective: Chronic Achilles tendon rupture can lead to decreased ankle joint plantar flexion and gait impairment. Open 1 or 2 incision flexor hallux longus transposition has been shown to have good functional results, but the disadvantage is that the operation is relatively extensive in the vulnerable site. To reduce the risk of soft tissue problems, flexor longus tendon transfers can be performed endoscopically.
\end{abstract}

Methods: An endoscopic transposition of flexor hallux longus was introduced.

Results: There were no wound healing problems or infection. Five of the six patients had a single-leg heel elevation on the affected side 12 months after surgery.

Conclusion: The functional results are good. Soft tissue dissection was minimal and no patients had postoperative wound healing problems or infection. Endoscopic flexor hallux longus transfer may be a surgical approach and may also be considered in patients with underlying wound healing problems. Level of Evidence: Level 4: Technical Instructions/ Uncontrolled Case Series

Keywords: foot surgery techniques, diagnostic and therapeutic techniques, Achilles tendinitis, surgical complications, unstable ankle joint
Volume 6 Issue I - 202 I

\author{
Chao Zhou,' Honghai Yang,' Hua Zhou,' \\ Feina Liu, 'Yan Zheng, ${ }^{2}$ Yanhong Bi,' Xin \\ Wang, ${ }^{2}$ Zhanzhen $\mathrm{Li}^{2}$ \\ 'Department of Foot and Ankle, Ding HaiGuanghua hospital, \\ China \\ ${ }^{2}$ Department of Orthopedic, Ding HaiGuanghua hospital, China
}

Correspondence: Xin Wang, Orthopedic Department, Ding HaiGuanghua hospital, No.468 Huifei Road, Zhoushan City, Zhejiang Province, China, 316000, Tel +860 l3522263 I03, Fax +86-0580-29800 I I, Email wangx|26@I26.com

Zhan zhen Li, Orthopedic Department, Ding HaiGuanghua hospital, No.468 Huifei Road, Zhoushan City, Zhejiang Province, China, 316000, Tel +860I 3505802688, Fax +86-0580-29800II, Email lizz2688@163.com

Received: January 02, 202I | Published: January 27, 2021

\section{Introduction}

This is a method of restoring structural continuity and has advantages over other methods because it may overlap large gaps and partially or completely replace the plantar flexion of the ankle. In addition, the muscles of FHL can provide a certain amount of blood vessels for the degenerated Achilles tendon and promote adjacent healing. A series of 2 patients demonstrated the good effect of flexor longus tendon (FHL) transplantation for the treatment of chronic Achilles tendon rupture. Results: The American Orthopaedic Foot and Ankle Joint (AOFAS) score was about 90 after FHL transplantation. Traditionally, FHL transplantation has been performed with a single postero medial incision or two-incision technique; Postero medial and plantar, remove the tendon from outside the Henry node. When open surgery is performed, the scope of the operation may be quite extensive and located in vulnerable areas. ${ }^{1-3}$ In order to reduce surgical trauma and thus possible soft tissue healing problems, we introduced the endoscopic technique of transferring FHL for CATR. This article introduces the technique and early experience of endoscopic treatment of FHL in CATR. Similar techniques have been described by Goncalves et al. Hong et al, ${ }^{4}$ and Gossage et al. ${ }^{5}$ in 1,4 and 2 patients, respectively. No other publication describes the endoscopic technique of FHL transfer CATR.

\section{Materials and methods}

From February to September 2015, six patients (1 female, 5 male) underwent endoscopic FHL metastases. Complete ruptures were confirmed in all patients and all patients underwent preoperative magnetic resonance imaging (MRI) scans. All ruptures of Achilles tendon were confirmed clinically and by MRI. None of the patients had surgery on the Achilles tendon or had steroid injections prior to rupture.

The study was approved by data protection officers at University Hospital. The patient has signed an informed consent. The operation was performed in a center by two experienced ankle and foot orthopedic surgeons.

\section{Indications and contraindications}

CATR was defined as any rerupture of the Achilles tendon and was ignored or diagnosed more than 6 weeks after rupture. We also included ruptures where conservative or surgical treatment failed, and where intensive physical therapy failed to restore function to the patient's satisfactory level, the rupture healed, function decreased, and the Achilles tendon was significantly prolonged. In addition, FHL transplantation under endoscopy can be considered for patients with severe Achilles tendon degeneration accompanied by acute Achilles tendon rupture.

When a soft tissue wound, blister, or persistent infection occurs in the portal vein placement site, the patient will not undergo surgery or postpone surgery until the soft tissue is acceptable.

Endoscopic FHL metastasis will also be considered if the general health of elderly patients, patients with diabetes, or patients with other underlying risk factors for soft tissue healing does not represent an unacceptable risk for surgery. None of the patients were smokers at the time of the operation. In order to have the surgery, all patients must be non smokers or should quit smoking before the surgery.

\section{Preoperative plan}

The patient was operated under general anesthesia. The limbs were prepared with chlor hexidinsprit farget ethanol solution (Klor hexidinsprit Farget $5 \mathrm{mg} / \mathrm{mL}$, Fresenius Kabi Norge A /S, Oslo, Norway). Prepare both limbs on the operating table to compare tendon tension during the perio perative period and to ensure more plantar flexion on the operating side. Place the patient in the prone position and apply a barometric tourniquet on the ipsilateral thigh at a pressure of $250 \mathrm{mmHg}$. The foot is placed on a mat at the edge of the table to allow the ankle dorsiflexion. 


\section{Surgical techniques}

Make the posterior lateral and posterior inner portals according to the procedure described by Van Dijk et al. ${ }^{6}$ A $4.5 \mathrm{~mm}$ arthroscopy was introduced through a postero lateral incision (Smith \& Nephew, Andover, MA, USA), and a $4.5 \mathrm{~mm}$ razor $(4.5 \mathrm{~mm}$ incisor with Elite blade, Dynomics arthroscopic surgical blade, Smith \& Nephew, Andover, MA, USA) was introduced through the postero medial entrance. The razor is introduced medially to laterally, carefully keeping the instrument on the posterior neurovascular bundle and lateral to the FHL tendon. Identify the FHL tendon. The deep calf fascia, located behind the FHL tendon, is divided into tendons lengthways proximally, from the distal edge of the lateral margin of the FHL tendon to about $5 \mathrm{~cm}$ above the talocral joint. This is done to reduce the Angle of the FHL tendon when the tendon is transferred to the calcaneal tubercle. The flexor retinal is cut along its lateral margin to the bone to open distally and reveal the FHL tendon as far as possible (Figure A). After the preparation of the FHL tendon, prepare the area of the anterior calcaneal tuberosity of the Achilles tendon. Through a proximal accessory posterior medial gate, a wire is drilled into the calcaneus and points to the midline slightly lateral to the underside of the foot, behind and near the Achilles tendon. The position was controlled by fluoroscopy. The bone tunnel was drilled with a $7 \mathrm{~mm}$ hollow drill, and a plantar incision was made at the bone tunnel. After the preparation of the FHL tendon and bone tunnel, the ankle joint and the hallux were plantar flexed; The FHL tendon is tensioned and severed endosco pically as far as possible within the fibrous bone tunnel. Care must be taken to maintain the medial side of the FHL tendon to avoid injury to the neurovascular bundle. If necessary, an auxiliary proximal portal vein can be made to clamp the FHL tendon. The proximal FHL tendon stump was pulled out through the posterior medial gate and propped with a needle before the temporary stay suture was applied (Figure B). The tendon stump was reintroduced with temporary stay suture to the rear foot.

The suture retriever is introduced through the bone tunnel from the plantar of the calcaneus. Clamps can be used to help secure stitches. The FHL tendon is pulled from the inside out through the bone tunnel of the calcaneal tubercle until the FHL muscle is located at the entrance of the bone tunnel. The tension on the operating side is slightly higher. An interference screw (RCI screw, $7 \mathrm{~mm} \times 35 \mathrm{~mm}, 8 \mathrm{~mm}$ screw head, Smith \& Nephew Inc., Andover, MA, USA) was introduced into the bone tunnel with a guide wire, and the ankle plantar flexed were bent to anchor the FHL tendon under tension (Figure C). The tension was compared with that of the contralateral ankle joint. The gate and the wound were closed (Figure D).

The operative limb was placed in a splint below the knee with the ankle flexed at $30^{\circ}$ for 2 weeks and then placed in a cast below the knee with the ankle in neutral position. After 2 weeks, loading was allowed in the prefabricated AFO (Novawalk, Jhinova AB, Hasselby, Sweden) and the insole height was reduced for the next 8 weeks. Rehabilitation training under the guidance of physiotherapists began 4 weeks after surgery.

\section{Results}

The median age was 61 years (51-73 years). Patients were classified according to the American Society of Anesthesiologists (ASA). One patient was classified as ASA 1 and three were classified as ASA 2 and two were classified as ASA 3. Two cases were diagnosed with late Achilles tendon rupture (surgery 12-13 months after rupture), and four cases were treated conservatively (surgery 12-21 months after rupture), with increased ankle dorsal flexion, significantly reduced plantar flexion, and decreased walking ability, even after intensive training with movement assistance by a physical therapist. All patients maintained tendon continuity preoperatively. After FHL tendon grafts, all patients had increased volume in the posterior part of the ankle and in the medial Achilles tendon associated with FHL, but this nerve damage was temporary and did not occur at 12 months follow-up. One patient developed plantar heel pain 1 year after FHL transplantation, but this did not appear to be related to the operation. All patients were followed up at the outpatient clinic for at least 12 months. All patients showed better function and increased plantar flexion strength at 12 months postoperatively, but still had reduced plantar flexion strength compared to the unaffected side. Three months after surgery, none of the patients could perform a single foot toe lifting test on the affected ankle. All patients with the exception of 1 underwent unilateral foot elevation at 12 months postoperatively. At 2 years of follow-up, the patient had a lengthened tendon graft, increased ankle dorsiflexion, and ectopic bone formation at the location where the interference screw was inserted. No rupture of metastatic FHL was recorded.

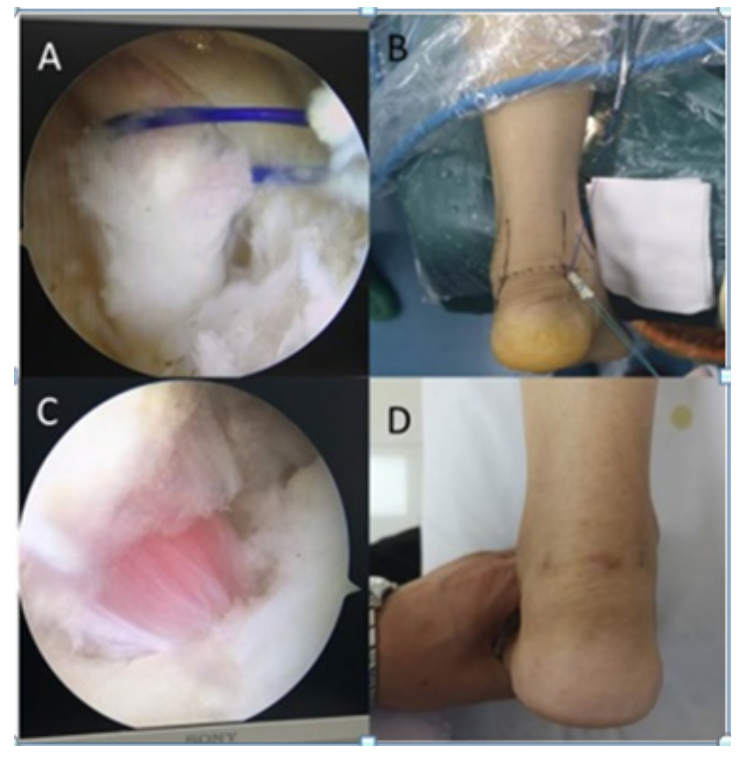

Figure 1 A. Identify the FHL tendon and the FHL tendon is tensioned and cut under endoscopic vision.

B. The proximal FHL tendon stump is pulled out through the posteromedial portal

C. Anchor the FHL tendon in the tunnel hole with tension

D. post operative appearance

\section{Discussion}

We introduced the surgical method of endoscopic FHL transplantation for the treatment of chronic Achilles tendon rupture, and combined with the early experience of 6 patients who underwent this method in our hospital. Several surgical approaches and combinations of different techniques have been used to repair CATR. Direct end-to-end suturing is due to prolonged retraction of the tendon stump, or repair of CATR is neither recommended nor possible when a large amount of degenerative tendon tissue is present. $\mathrm{V}-\mathrm{Y}$ plastic surgery has the advantage of maintaining the continuity of the gastric soleus complex and can be performed in the case of medium size defects $(2-5 \mathrm{~cm}) .17-19$ However, there have been some reports of $8-10 \mathrm{~cm}$ defects being closed by this method. 20 Other Achilles tendonofascial inversion procedures, such as Lindholm 
plastic surgery, are available and described as being able to handle a 5 to $9 \mathrm{~cm}$ tendon space. 21,22 Larger gaps can also be managed by free tendon grafting. 12 The disadvantage of using these procedures is that they involve a large soft-tissue dissection, resulting in a loss of flow of a portion of the tendon tissue, and may pose a threat to wound healing and surgical site infection. El Shewy et al. ${ }^{7}$ described 5 out of 9 patients who had wound healing problems and local infection after surgery with the gastrocemius soleus complex fascia flap. Open FHL metastasis, including two-incision and single-incision techniques, is a surgical approach that may require extensive surgery at vulnerable sites. In a retrospective patient series, Alhaug et al. ${ }^{8}$ described local infection and/or wound healing problems in 7 of 17 patients (only non-smokers) who underwent the 2-incision FHL transfer technique. Hahn et al. ${ }^{9}$ reported wound healing problems in 4 of 16 patients and neurological complications in 2 . Rahm et al. ${ }^{10}$ reported infection or wound healing problems in 7 of 42 patients $(17 \%)$. We performed surgery on 6 patients with endoscopic FHL metastasis and had no infection or wound healing problems, as did the 4 patients described by Hong et al. ${ }^{4}$ patients underwent FHL metastasis using a similar endoscopic technique. Results after endoscopic FHL metastasis are promising in terms of soft tissue complications; however, the total number of patients described in our paper, together with Hong et al. ${ }^{4}$ Goncalves et al. Gossage et al. ${ }^{5}$ are low. Endoscopy is attractive because of its merits as a minimally invasive procedure, but it is also technically demanding. Many surgeons do not use open techniques for surgical treatment of patients with impaired wound healing or an increased risk of infection. The lower incidence of soft tissue complications associated with endoscopic techniques may lead to the use of this method also for patients with underlying wound healing problems. In the current patient profile, 2 out of 6 patients had damage to the medial calcaneal nerve, although it was temporary. This means careful endoscopic dissection and cutting of the FHL tendon should be done with care.

Nerve damage after endoscopic FHL metastasis has not been mentioned in previous reports. 13 -- 15 Herbst et al. ${ }^{11} 24$ reported injury to the medial plantarsal nerve using the two-incision technique. Monroe et al. 8 reported that initial partial sensory loss occurred in 3 of 7 patients using the 2-incision technique. To ensure adequate length of the FHL tendon, the ankle and big toe must be bent on the sole of the foot when the FHL tendon is cut. Care must be taken to avoid soft tissue interference with the tendon when moving the tendon from the portal vein into the bone tunnel after placing the fixed sutures. To avoid the tendons of the tent, the fascia of the foot is separated about 5 $\mathrm{cm}$ below the proximal end of the FHL. Hong et al. ${ }^{4}$ Goncalves et al. or Gossage et al. ${ }^{5}$ do not describe this. For better plantar flexion, place the calcaneal tunnel far back and close to the Achilles tendon to increase arm weight. 25 Arastu et al. ${ }^{12} 25$ In a computer program study, it was demonstrated that the posterior positioning of the FHL tendon on the nodule increases the strength of the calcaneus but reduces the range of motion of the ankle. Furthermore, in our experience, the FHL tendon should be pulled into the bone tunnel so that the FHL muscle almost enters the bone tunnel. The assessment of additional tension should be compared with that of the contra lateral ankle. Temporary clamps can be placed on the sole of the foot to keep the tendons tight. Therefore, it is advisable to prepare legs on the operating table. In the 2-incision technique, the tendon is pulled through a medial to lateral calcaneal tunnel to form a ring and is sutured to itself and/or the proximal stump of the Achilles tendon. 6,26 For the endoscopic technique, the tendon is fixed to the bone using interference screws. Similarly, for the single-incision technique of Achilles tendon transfer, the interference screw anchorage of the FHL tendon can be used. Tendon after 7, 27 interfering with screws, and by introducing FHL tendons open calcaneal bone tunnel incision surgery compared with 2, using the interference screws of plantar flexion force or functional outcome after tendon is circular and annular bodies in a recent study, with their own suture in the ultimate strength, peak stress or damage strain has no difference. 28 A recent cadaver study investigated the failure loads of suture anchoring compared with Biotenodesis screws for calcaneal FHL tendon fixation. In younger specimens, the Biotenodesis screw showed a greater destructive load than the anchor. 29 Because the calcaneus has a soft bone structure, we selected a bone tunnel with the same diameter as the interfering screw and used a relatively large diameter compared to the size of the transferred tendon. DeCarbo et al. ${ }^{13}$ recommended a $0.5 \mathrm{~mm}$ ream in addition to the planned diameter of the interference screw to reduce the risk of screw fracture or metastatic FHL tendon fragmentation. The diameter of the FHL tendon is 4 to $5 \mathrm{~mm}$. 5 Another problem with the endoscopic FHL transplantation technique is that degenerated tendon tissue is not removed and the tendon graft is not supplemented. However, Wapner et al. ${ }^{14}$ supported repair with FHL transplantation alone and found better results in patients who did not receive any supplements. Tendon grafts have the advantage of bringing well-vascularized tissue into the area to provide healing. 1,2 Hahn et al. ${ }^{9}$ suggest retaining at least part of the Achilles tendon to control length. Three months after surgery, none of the patients could perform a single foot elevation test on the affected side. One year after the operation, except for 1 patient, the other patients with lateral heel elevation. Patients thought that the elution was significantly better than the other side, but still weaker than the other side. In the current patient series, no comprehensive functional test is planned to assess the strength and endurance of plantar flexion. In most studies, the planar flexion force after FHL transfer is less than $25 \%$ to $35 \%$ compared to the other leg. 3,5-8 Plantar flexion of $30^{\circ}$ 2 weeks postoperatively, followed by a neutral ankle position for the next 2 weeks, may be an overly aggressive approach with respect to the range of motion of the ankle, which may result in too long tendon structures posterior. Our experience led us to change the postoperative procedure so that AFO with insole was performed 8 weeks after 2 weeks, with no neutral ankle position. The physiotherapist and patient must be informed to postpone stretching. ${ }^{15-23}$

\section{Conclusion}

Experience with FHL displacement with chronic Achilles tendon rupture is good, but the experience with endoscopic techniques for FHL displacement is limited. However, endoscopic FHL metastasis involves minimal soft tissue dissection, and therefore the risk of soft tissue healing problems is low. The functional results in this series of cases are promising, and since soft tissue dissection is small, it may be a surgical approach that may be considered for patients with underlying wound healing problems. However, prospective studies involving more patients are necessary to assess functional outcomes and confirm lower wound healing and infection rates.

\section{Funding}

None.

\section{Acknowledgments}

None.

\section{Conflicts of interest}

The authors declare no conflicts of interest. 


\section{References}

1. Padanilam TG. Chronic achilles tendon ruptures. Foot Ankle Clin. 2009;14:711-728.

2. Kraeutler MJ, Purcell JM, Hunt KJ. Chronic achilles tendon ruptures. Foot Ankle Int. 2017;38:921-929.

3. Leitner A, Voigt C, Rahmanzadeh R. Treatment of extensive aseptic defects in old Achilles tendon ruptures: methods and case reports. Foot Ankle. 1992;13:176-180.

4. Hong CC, Nag K, Tan KJ. Arthroscopic flexor hallucislongus transfer for chronic Achilles tendon rupture. Tech Foot Ankle Surg. 2015;14:128133.

5. Gossage W, Kohls-Gatzoulis J, Solan M. Endoscopic assisted repair of chronic Achilles tendon rupture with flexor hallucislongus augmentation. Foot Ankle Int. 2010;31:343-347.

6. van Dijk CN, Scholten PE, Krips R. A 2-portal endoscopic approach for diagnosis and treatment of posterior ankle pathology. Arthroscopy. 2000;16:871-876.

7. Mohamed Taha, El Shewy, Hassan Magdy, et al The american journal of sports medicine. 2009;37(8):1570-1577.

8. Alhaug OK BG, Husebye EE, Hvaal KH. Transposis jonav fleksor hallucislon gusved kronis kakilless eneruptur. Paper presented at: The Annual Norwegian Orthopaedic Meeting; 2014.

9. Hahn F, Meyer P, Maiwald C, et al. Treatment of chronic Achilles tendinopathy and ruptures with flexor hallucis tendon transfer: clinical outcome and MRI findings. Foot Ankle Int. 2008;29:794-802.

10. Stefan, Rahm, Christian, Spross, Fabienne, Operative treatment of chronic irreparable achilles tendon ruptures with large flexor hallucis longus tendon transfers. Foot \& ankle international. 2013;34;8:11001110 .

11. Herbst SA, Miller SD. Transection of the medial plantar nerve and hallux cock-up deformity after flexor hallucislongus tendon transfer for Achilles tendinitis: case report. Foot Ankle Int. 2006;27:639- 641.
12. Arastu MH, Partridge R, Crocombe A, et al. Determination of optimal screw positioning in flexor hallucislongus tendon transfer for chronic tendoachilles rupture. Foot Ankle Surg. 2011;17:74-78.

13. DeCarbo WT, Hyer CF. Interference screw fixation for flexor hallucislongus tendon transfer for chronic Achilles tendonopathy. J Foot Ankle Surg. 2008;47:69-72.

14. Wapner KL, Pavlock GS, Hecht PJ, et al. Repair of chronic Achilles tendon rupture with flexor hallucislongus tendon transfer. Foot Ankle. $1993 ; 14: 443-449$.

15. Coull R, Flavin R, Stephens MM. Flexor hallucislongus tendon transfer: evaluation of postoperative morbidity. Foot Ankle Int. 2003;24:931-934.

16. Myerson MS. Management of non insertional tendinopathy. In: reconstructive foot and ankle surgery: management of complications. 2nd ed. Philadelphia, PA: Elsevier Saunders; 2010:334-335.

17. Den Hartog BD. Flexor hallucislongus transfer for chronic Achilles tendonosis. Foot Ankle Int. 2003;24:233-237.

18. Lee JI. Endoscopic flexor hallucis longus tendon transfer for reconstruction of the achilles tendon rupture in high-risk patients: a case series. J Foot Ankle Surg. 2019 Nov;58(6):1257-1261.

19. Kissel CG, Blacklidge DK, Crowley DL. Repair of neglected Achilles tendon ruptures-procedure and functional results. J Foot Ankle Surg. 1994;33:46-52.

20. Park YS, Sung KS. Surgical reconstruction of chronic Achilles tendon rupturesusing various methods. Orthopedics. 2012;35:e213-e218.

21. Wilcox DK, Bohay DR, Anderson JG. Treatment of chronic Achilles tendon disorders with flexor hallucislongus tendon transfer/ augmentation. Foot Ankle Int. 2000;21:1004-1010.

22. Liu GT, Balldin BC, Zide JR, et al. A biomechanical analysis of interference screw versus bone tunnel fixation of flexor hallucislongus tendon transfers to the calcaneus. J Foot Ankle Surg. 2017;56:813-816.

23. Drakos MC, Gott M, Karnovsky SC, et al. Biomechanical analysis of suture anchor vstenodesis screw for FHL transfer. Foot Ankle Int 2017;38:797-801. 